Forthcoming in Philosophical Studies

\title{
Accuracy-Dominance and Conditionalization
}

\author{
Michael Nielsen
}

January 26, 2021

\begin{abstract}
Epistemic decision theory produces arguments with both normative and mathematical premises. I begin by arguing that philosophers should care about whether the mathematical premises (1) are true, (2) are strong, and (3) admit simple proofs. I then discuss a theorem that Briggs and Pettigrew (2020) use as a premise in a novel accuracy-dominance argument for conditionalization. I argue that the theorem and its proof can be improved in a number of ways. First, I present a counterexample that shows that one of the theorem's claims is false. As a result of this, Briggs and Pettigrew's argument for conditionalization is unsound. I go on to explore how a sound accuracy-dominance argument for conditionalization might be recovered. In the course of doing this, I prove two new theorems that correct and strengthen the result reported by Briggs and Pettigrew. I show how my results can be combined with various normative premises to produce sound arguments for conditionalization. I also show that my results can be used to support normative conclusions that are stronger than the one that Briggs and Pettigrew's argument supports. Finally, I show that Briggs and Pettigrew's proofs can be simplified considerably.
\end{abstract}

Keywords. Accuracy; conditionalization; credal acts; credal strategies; dominance; epistemic decision theory; learning; probability; updating

\section{Introduction}

Epistemic decision theory is a research program that defends certain epistemic norms by arguing that they promote accuracy or proximity to the truth. There are two types of premises in these arguments. First, there are normative premises. These say something to the effect of: Agents are required to have epistemic states that are as accurate as possible. Second, there are mathematical premises. These say something to the effect of: All and only those epistemic states satisfying such-and-such properties are as accurate as possible. Combining these two types of premises yields norms to the effect of: Agents are required to have epistemic states that satisfy such-and-such properties.

This presentation of epistemic decision theory's arguments is schematic, of course. In order to produce substantive normative conclusions, one must specify which epistemic states one is interested in and what it means to be as accurate as possible. In this paper, I will be interested in credences and plans for updating credences in response to evidence. ${ }^{1}$ The question remains: What does it mean to be as accurate as possible?

\footnotetext{
${ }^{1}$ Below, I refer to the latter as credal acts.
} 
There are two standard answers in the epistemic decision theory literature: one answer given by those defending norms for credences and another answer given by those defending norms for updating plans. According to the standard defense of probabilism - a norm that says it's impermissible for agents to have credences that do not satisfy the probability axioms - a credence function is as accurate as possible if it is not dominated - if there is no alternative credence function that is more accurate in every possible world. According to the standard defense of conditionalization - a norm that says agents should plan to update their credences by adopting conditional probabilities given the evidence - an updating plan is as accurate as possible if it minimizes expected inaccuracy relative to an agent's probabilistic credence function - that is, if there is no alternative updating plan that the agent expects to be more accurate.

In a recent paper, Briggs and Pettigrew (2020) have argued that the expected inaccuracy argument for conditionalization has a number of shortcomings. They argue, for instance, that the expected inaccuracy argument shows that violating conditionalization is akin to choosing an act with lower expected utility than some other available act. But, although it's plausible that agents who make choices like this are unreasonable, it's not clear that they are inconsistent - an act with low expected utility might end up paying handsomely, after all. On the other hand, agents who choose dominated acts choose to suffer what are, by their own lights, sure losses. This looks much more like a form of inconsistency. So, insofar as conditionalization is meant to be a norm of consistency, as some have claimed (Armendt, 1992; Christensen, 1991, 1996; Lewis, 1999), it is important to investigate whether it can be defended with a dominance argument. ${ }^{2}$

Briggs and Pettigrew have made significant progress on this investigation, producing an argument that conditionalization uniquely avoids a certain kind of accuracy-dominance. I will show, however, that their argument is flawed. My focus will be on Briggs and Pettigrew's mathematical premises, which they present as a theorem. I will not dispute their normative premises (though I will consider what conclusions alternative normative premises support).

Why should philosophers interested in epistemic decision theory care about mathematical premises? After all, they do not contain normative, or otherwise philosophical, content. Isn't an analysis of a mathematical premise a merely mathematical exercise?

I think not, for several reasons. First and foremost, philosophers should care about whether mathematical premises are true. This is obvious. Only true premises - mathematical or otherwise - can play a role in sound arguments for normative conclusions. Second, philosophers should be concerned with establishing mathematical premises that are strong. Stronger mathematical premises support stronger normative conclusions; they help to tell us more about what kinds of epistemic states agents are required to have. Third, and perhaps less obviously, philosophers should be concerned with providing simple proofs of the mathematical claims that figure into their arguments. One reason this is true is that simple proofs are easier to generalize, and mathematical generalization can be philosophically important. An example of this can be found within epistemic decision theory itself. A number of recent papers (Carr, 2019; Gallow, 2019; Schoenfield, 2017) have argued that the expected inaccuracy argument for conditionalization, due to Greaves and Wallace (2006), makes assumptions about the structure of evidence that are implausibly strong. In particular, Greaves and Wallace assume that evidential propositions form a partition, and numerous examples show that

\footnotetext{
${ }^{2}$ Another shortcoming of the expected inaccuracy argument is mentioned in Section 3.
} 
this need not always be the case. In order to generalize Greaves and Wallace's argument for conditionalization, then, it's necessary to generalize the theorem underwriting it; it's necessary to investigate what kinds of updating plans minimize expected inaccuracy without the assumption that evidence forms a partition. I anticipate the same dialectic unfolding in the accuracy-dominance framework. While I, following Briggs and Pettigrew, will assume for now that evidence forms a partition, I recognize that this assumption is not really philosophically defensible. Ultimately, it should be relaxed, and the theorems that I present below should be generalized. Future work on this project will be facilitated by avoiding unnecessarily complicated mathematical machinery.

Echoing the three points I just made, my contributions in this paper can be summarized as follows. First, I will show that one of the claims in Briggs and Pettigrew's theorem is false. As a result, their argument for conditionalization is unsound. I will go on to explore how a sound accuracy-dominance argument for conditionalization might be recovered. In doing so, I will prove two new results that strengthen and correct the Briggs and Pettigrew theorem in various ways. In addition to supporting the conclusion that Briggs and Pettigrew argue for, my results also support normative conclusions that are stronger than the one supported by Briggs and Pettigrew's argument. Finally, I will show that the heavy mathematics used in Briggs and Pettigrew's proof (e.g. a transfinite induction, transfinite versions of several theorems from real analysis, and an appeal to the axiom of choice) can be eliminated. My hope is that the comparatively simple proofs offered here can be generalized in future work.

The rest of the paper is organized as follows. Section 2 contains a more detailed presentation of some of the arguments mentioned in this introduction and lays the groundwork for what follows. Section 3 contains the paper's main contributions. There, I present a counterexample to a claim in Briggs and Pettigrew's theorem, as well as two theorems that correct and improve Briggs and Pettigrew's theorem. I demonstrate the philosophical significance of my theorems by showing that they support several interesting normative conclusions. Section 4 summarizes my arguments, and two appendices contain the proofs of my theorems.

\section{Preliminaries and Background}

This section will follow Briggs and Pettigrew (2020) closely.

\subsection{Credence Functions and Inaccuracy Measures}

Let $\Omega$ be a finite set of possible worlds, and let $\mathcal{A}$ be an algebra of subsets of $\Omega$, or propositions. Without any real loss of generality, I will assume that $\mathcal{A}$ is the powerset of $\Omega$.

A credence function $c: \mathcal{A} \rightarrow[0,1]$ is a function that assigns a number in the closed unit interval to each proposition in $\mathcal{A}$. Let $\mathcal{C}$ be the set of all credence functions. A credence function is probabilistic if it obeys the standard axioms of probability theory.

An example of a probabilistic credence function is the valuation function $v_{\omega}$ at $\omega$, which is defined for all $\omega \in \Omega$ and $A \in \mathcal{A}$ by

$$
v_{\omega}(A)=\left\{\begin{array}{l}
1 \text { if } \omega \in A ; \\
0 \text { if } \omega \notin A .
\end{array}\right.
$$


The valuation function at $\omega$ assigns credence 1 to propositions that are true at $\omega$ and credence 0 to propositions that are false at $\omega$.

An inaccuracy measure $\mathcal{J}: \Omega \times \mathcal{C} \rightarrow[0, \infty]$ is defined initially to be a function that assigns a non-negative number in the extended real line to each pair of possible worlds and credence functions. $\mathcal{J}_{\omega}(c)$ is a numerical representation of $c$ 's inaccuracy in world $\omega$. Later on, we will extend the domain of $\mathcal{J}$.

It's standard to assume that $\mathcal{J}$ has the following properties. The only property that will be used explicitly in this paper is the last one, but the others play a role in the background results of Predd et al. (2009) and Briggs and Pettigrew (2020).

Separability There exists a function $i: \Omega \times \mathcal{C} \times \mathcal{A} \rightarrow[0, \infty]$ such that

$$
\mathcal{J}_{\omega}(c)=\sum_{A \in \mathcal{A}} i_{\omega}(c, A)
$$

for all $\omega \in \Omega, c \in \mathcal{C}$, and $A \in \mathcal{A}$. Think of $i_{\omega}(c, A)$ as the inaccuracy of $c$ at $\omega$ for a particular proposition $A$. Separability says that the overall inaccuracy $\mathcal{J}_{\omega}(c)$ of $c$ at $\omega$ is the sum of $c$ 's inaccuracies at $\omega$ for propositions.

Continuity For all $\omega \in \Omega, i_{\omega}(c, A)$ is a continuous function of $c(A)$. That is, if $\left(c_{n}\right) \in \mathcal{C}$, $\left(A_{n}\right) \in \mathcal{A}$, and the sequence $\left(c_{n}\left(A_{n}\right)\right)$ of real numbers converges to $c(A)$ for some $c \in \mathcal{C}$ and $A \in \mathcal{A}$, then $\lim _{n \rightarrow \infty} i_{\omega}\left(c_{n}, A_{n}\right)=i_{\omega}(c, A)$.

Extensionality For all $\omega, \omega^{\prime} \in \Omega, c, c^{\prime} \in \mathcal{C}$, and $A, A^{\prime} \in \mathcal{A}$, if $v_{\omega}(A)=v_{\omega^{\prime}}\left(A^{\prime}\right)$ and $c(A)=$ $c^{\prime}\left(A^{\prime}\right)$, then $i_{\omega}(c, A)=i_{\omega^{\prime}}\left(c^{\prime}, A^{\prime}\right)$. This says that the inaccuracy of $c$ at $\omega$ for the proposition $A$ is determined entirely by $A$ 's truth value at $\omega$ and the credence that $c$ assigns to $A$.

Strict Propriety If $c$ is a probabilistic credence function and $c^{\prime} \neq c$ is a credence function, then

$$
\sum_{\omega \in \Omega} c\{\omega\} \mathcal{J}_{\omega}(c)<\sum_{\omega \in \Omega} c\{\omega\} \mathcal{J}_{\omega}\left(c^{\prime}\right)
$$

This says that $c$ is the unique minimizer of $c$-expected inaccuracy.

One widely discussed inaccuracy measure satisfying Separability, Continuity, Extensionality, and Strict Propriety is the Brier score, defined for all $\omega \in \Omega$ and $c \in \mathcal{C}$ by

$$
\mathcal{J}_{\omega}(c)=\sum_{A \in \mathcal{A}}\left(v_{\omega}(A)-c(A)\right)^{2}
$$

I'll use the Brier score a bit later in the paper as part of a counterexample.

I now define two ways in which one credence function can accuracy-dominate another.

Strong Accuracy-Dominance Let $c, c^{\prime} \in \mathcal{C}$. Then, $c^{\prime}$ strongly accuracy-dominates $c$ iff $\mathcal{J}_{\omega}\left(c^{\prime}\right)<\mathcal{J}_{\omega}(c)$ for all $\omega \in \Omega$.

Weak Accuracy-Dominance Let $c, c^{\prime} \in \mathcal{C}$. Then, $c^{\prime}$ weakly accuracy-dominates $c$ iff 
- $\mathcal{J}_{\omega}\left(c^{\prime}\right) \leq \mathcal{J}_{\omega}(c)$ for all $\omega \in \Omega$, and

- $\mathcal{J}_{\omega}\left(c^{\prime}\right)<\mathcal{J}_{\omega}(c)$ for some $\omega \in \Omega$.

So, $c^{\prime}$ strongly accuracy-dominates $c$ if it is strictly less inaccurate than $c$ in every possible world, and it weakly accuracy dominates $c$ if it is no more inaccurate than $c$ in every possible world and strictly less inaccurate than $c$ in some possible world.

My focus in this paper will be accuracy-dominance theorems concerning conditionalization, but I will have occasion to appeal to the following widely-discussed result concerning probabilism. The result shows that every non-probabilistic credence function is strongly accuracy-dominated by some probabilistic credence function, whereas probabilistic credence functions are not even weakly accuracy-dominated.

Theorem 1 (Predd et al. (2009), Theorem 1). Let c be a credence function, and assume that $\mathcal{J}$ satisfies Separability, Continuity, Extensionality, and Strict Propriety. Then,

- If $c$ is probabilistic, then there is no credence function that weakly accuracy-dominates it.

- If $c$ is not probabilistic, then there is some probabilistic credence function that strongly accuracy-dominates it.

Note how Theorem 1 can be coupled with a normative premise to yield an argument for probabilism.

Normative Premise for Probabilism It's impermissible for an agent to adopt a credence function $c$ if there is another credence function that (i) strongly accuracy-dominates $c$, and (ii) is not itself weakly accuracy-dominated.

Assuming the inaccuracy measure satisfies the relevant properties, this normative premise and Theorem 1 imply that it is impermissible for an agent to adopt a credence function that is not probabilistic.

\subsection{Credal Acts, Expected Inaccuracy, and Conditionalization}

In this subsection, I'll begin by introducing credal acts and conditionalization. Then I'll present the Greaves and Wallace (2006) result, which shows that conditionalization uniquely maximizes expected inaccuracy.

An evidence partition $\mathcal{E}=\left\{E_{1}, \ldots, E_{n}\right\}$ is a pairwise disjoint subset of $\mathcal{A}$ whose union is $\Omega$. For all $\omega \in \Omega$, let $\mathcal{E}_{\omega}$ be the unique member of $\mathcal{E}$ that contains $\omega$. Think of $\mathcal{E}$ as representing the possible outcomes of an experiment that an agent is about to perform. If $\omega$ is the actual world, then the agent will learn $\mathcal{E}_{\omega}$.

A credal act $a: \mathcal{E} \rightarrow \mathcal{C}$ is a function that maps propositions in the evidence partition to credence functions. Think of the value of $a$ at $E \in \mathcal{E}$, denoted by $a_{E}$, as the credence function that an agent plans to adopt if she learns $E$. We extend the inaccuracy measure of credence functions to credal acts by defining

$$
\mathcal{J}_{\omega}(a)=\mathcal{J}_{\omega}\left(a_{\mathcal{E}_{\omega}}\right) .
$$

This says that the inaccuracy of a credal act $a$ at world $\omega$ is equal to the inaccuracy of the credence function to which $a$ maps $\mathcal{E}_{\omega}$. 
I will be especially concerned with the following credal acts.

A conditionalization of a credence function $c$ is a credal act $a$ that satisfies

$$
c(A \cap E)=c(E) a_{E}(A)
$$

for all $A \in \mathcal{A}$ and $E \in \mathcal{E}$.

Note that the definition of conditionalization does not require $c$ to be probabilistic. If $c$ is probabilistic, however, and $c(E)>0$, then (3) is equivalent to

$$
a_{E}(A)=c(A \mid E) \text {. }
$$

In other words, conditionalizations of probabilistic credence functions are given by ordinary conditional probabilities, whenever the latter are well defined. If, on the other hand, $c$ is probabilistic and $c(E)=0$-or, more generally, if $c$ is any credence function and $c(E)=0=$ $c(A \cap E)$ - then any value of $a_{E}(A)$ satisfies $(3) ; a_{E}$ need not even be probabilistic in order to be a conditionalization of $c$ in cases like this. This fact, which I will return to momentarily, plays an important role in the argumentation of the next section.

But before we get to that, I need to present the expected accuracy argument for conditionalization. I begin by defining expected inaccuracy.

Let $c$ be a probabilistic credence function, and let $a$ be a credal act. Then, the $c$-expected inaccuracy of $a$ is defined as

$$
\exp _{c}(\mathcal{J}(a))=\sum_{\omega \in \Omega} c\{\omega\} \mathcal{J}_{\omega}(a)
$$

Greaves and Wallace show that, given a probabilistic credence function $c$, credal acts that are conditionalizations of $c$ uniquely minimize $c$-expected inaccuracy.

Theorem 2 (Greaves and Wallace (2006), Corollary 2). Let c be a probabilistic credence function, let a be a credal act, and assume that $\mathcal{J}$ satisfies Strict Propriety. Then, a minimizes c-expected inaccuracy if and only if $a$ is a conditionalization of $c$. In other words, if a is a conditionalization of $c$, then

$$
\exp _{c}(\mathcal{J}(a)) \leq \exp _{c}\left(\mathcal{J}\left(a^{\prime}\right)\right)
$$

for all credal acts $a^{\prime}$, and the inequality is strict unless $a^{\prime}$ is also a conditionalization of $c$.

Note how Theorem 2 can be coupled with a normative premise to yield an argument for conditionalization.

Normative Premise for Credal Act Conditionalization An agent with a probabilistic credence function $c$ is required to choose credal acts that minimize $c$-expected inaccuracy.

Assuming the inaccuracy measure satisfies Strict Propriety, this normative premise and Theorem 2 imply that agents with probabilistic credence functions are required to choose credal acts that are conditionalizations of their credence functions. 


\section{Accuracy-Dominance and Conditionalization}

In the introduction, I mentioned one shortcoming of the expected inaccuracy argument for conditionalization: it establishes only that conditionalization is a norm of reasonableness, not a norm of consistency. Having seen the argument in more detail, another shortcoming is now apparent: it applies only to agents who have probabilistic credence functions. The expected inaccuracy argument establishes the narrow-scope norm: an agent with a probabilistic credence function ought to [choose a credal act that is a conditionalization of her credence function]. It doesn't establish the wide-scope norm: an agent ought to [have a probabilistic credence function and choose a credal act that is a conditionalization of her credence function]. A further advantage of the accuracy-dominance argument for conditionalization-to be presented in this section - is that it doesn't assume that agents have probabilistic credence functions, and it can therefore be used to argue for the wide-scope norm. This is achieved formally by shifting our focus from credal acts to credal strategies, defined as follows.

A credal strategy $\langle c, a\rangle$ is a pair consisting of a credence function $c$ and a credal act $a$.

- $\langle c, a\rangle$ is probabilistic iff $c$ is probabilistic and $a_{E}$ is probabilistic for all $E \in \mathcal{E}$.

- $\langle c, a\rangle$ is a conditionalizing strategy iff $a$ is a conditionalization of $c$.

As mentioned above, much of my argument turns on the fact that conditionalizations can behave anomalously on propositions in the evidence partition that have zero credence. Let me emphasize this point by stating a simple but useful lemma about conditionalizing credal strategies. The proof of the lemma involves nothing more than applying the relevant definitions and is left as an exercise for the reader.

Lemma 1. Let $c$ be a probabilistic credence function, let $\langle c, a\rangle$ be a conditionalizing strategy, and let $E \in \mathcal{E}$.

(i) If $c(E)=0$ and $a^{\prime}$ is any credal act that agrees with a on propositions $E^{\prime} \in \mathcal{E}$ such that $E^{\prime} \neq E$, then $\left\langle c, a^{\prime}\right\rangle$ is also a conditionalizing strategy.

(ii) If, on the other hand, $c(E)>0$, then $a_{E}$ is uniquely determined by (3). Indeed, $a_{E}=$ $c(\cdot \mid E)$ and $a_{E}$ is probabilistic.

The first claim of Lemma 1 says that conditionalizations of probabilistic credence functions can behave arbitrarily on propositions in the evidence partition that get zero credence, while the second claim says that conditionalizations of probabilistic credence functions cannot behave arbitrarily on propositions in the evidence partition that get positive credence.

In order to extend the inaccuracy measure from credence functions and credal acts to credal strategies, Briggs and Pettigrew introduce a further assumption about $\mathcal{J}$.

Temporal Separability For all $\omega \in \Omega$ and all credal strategies $\langle c, a\rangle$,

$$
\mathcal{J}_{\omega}(\langle c, a\rangle)=\mathcal{J}_{\omega}(c)+\mathcal{J}_{\omega}(a) .^{3}
$$

\footnotetext{
${ }^{3}$ As Briggs and Pettigrew point out, Temporal Separability can be weakened to: There exist $\alpha, \beta \in(0, \infty)$ such that for all $\omega \in \Omega$ and all credal strategies $\langle c, a\rangle$,

$$
\mathcal{J}_{\omega}(\langle c, a\rangle)=\alpha \mathcal{J}_{\omega}(c)+\beta \mathcal{J}_{\omega}(a) .
$$
}


I can now present the theorem about accuracy-dominance and conditionalization that Briggs and Pettigrew (2020) report. Under the assumption that the inaccuracy measure satisfies Separability, Continuity, Extensionality, Strict Propriety, and Temporal Separability, Briggs and Pettigrew make the following three mathematical claims (Theorem 1, p. 171):

(I) For each credal strategy that is not probabilistic, there is an alternative credal strategy that is probabilistic and conditionalizing that weakly accuracy-dominates it.

(II) For each credal strategy that is not conditionalizing, there is an alternative credal strategy that is probabilistic and conditionalizing that strongly accuracy-dominates it.

(III) For each credal strategy that is probabilistic and conditionalizing, there is no alternative credal strategy whatsoever that even weakly accuracy-dominates it.

In response to these claims, I will argue for the following.

(a) (I) is true, but a stronger and more informative claim can be shown.

(b) (II) is true as well but admits a proof that is much simpler than the one that Briggs and Pettigrew give. In particular, the proof does not require the transfinite induction step in section 7.4 of Briggs and Pettigrew's paper.

(c) (III) is false, but a slightly weaker claim is true.

Let me begin by showing that (III) is false. The idea behind my counterexample to (III) is to consider a probabilistic credal strategy that assigns some proposition in the evidence partition zero credence. As pointed out above, in cases like this, there will be many conditionalizations of the given credence function, and they can behave quite arbitrarily. We can use this arbitrary behavior on zero credence propositions to define a probabilistic and conditionalizing credal strategy that is weakly accuracy-dominated. Here are the details.

\section{Counterexample to (III).}

- Let $\Omega=\left\{\omega_{1}, \omega_{2}, \omega_{3}\right\}$, and let $\mathcal{A}$ be the powerset of $\Omega$.

- Let $\mathcal{J}$ be the Brier score, defined by (1) and extended to credal strategies by (2) and Temporal Separability.

- Let $\mathcal{E}=\left\{\left\{\omega_{1}\right\},\left\{\omega_{2}, \omega_{3}\right\}\right\}$.

- Let $c\left\{\omega_{1}\right\}=0$ and $c\left\{\omega_{2}\right\}=1 / 2=c\left\{\omega_{3}\right\}$. Note that $c$ is probabilistic and assigns credence 0 to a proposition in the evidence partition.

- Let $a_{\left\{\omega_{1}\right\}}=v_{\omega_{2}}$ and $a_{\left\{\omega_{2}, \omega_{3}\right\}}=c\left(\cdot \mid\left\{\omega_{2}, \omega_{3}\right\}\right)$. That is, when the credal act $a$ learns $\left\{\omega_{1}\right\}$-a proposition with zero $c$-credence - it behaves quite anomalously and updates to the valuation function at $\omega_{2}$. When $a$ learns $\left\{\omega_{2}, \omega_{3}\right\}$, however, it updates to the $c$-conditional probability given $\left\{\omega_{2}, \omega_{3}\right\}$. Note that $a$ is a conditionalization of $c$, so the credal strategy $\langle c, a\rangle$ satisfies the antecedent of (III) - it is probabilistic and conditionalizing. 
- Finally, let $a_{\left\{\omega_{1}\right\}}^{\prime}=v_{\omega_{1}}$ and $a_{\left\{\omega_{2}, \omega_{3}\right\}}^{\prime}=c\left(\cdot \mid\left\{\omega_{2}, \omega_{3}\right\}\right)$. That is, the credal strategy $a^{\prime}$ behaves like $a$ on $\left\{\omega_{2}, \omega_{3}\right\}$ and updates to the $c$-conditional probability given $\left\{\omega_{2}, \omega_{3}\right\}$. On $\left\{\omega_{1}\right\}$, however, $a^{\prime}$ behaves less anomalously; instead of updating to the valuation function at $\omega_{2}, a^{\prime}$ updates to $v_{\omega_{1}}$. This seems less anomalous because it seems that if $a^{\prime}$ learns the proposition $\left\{\omega_{1}\right\}$, then $a^{\prime}$ should become certain of that proposition, which it does in this case. Note that $a^{\prime}$ is also a conditionalization of $c$ (Lemma 1(i)).

- To complete the counterexample, I claim that the credal strategy $\left\langle c, a^{\prime}\right\rangle$ weakly accuracydominates $\langle c, a\rangle$.

Proof of claim. Using Temporal Separability, the definition of $\mathcal{J}$ on credal acts, and the fact that $a_{\left\{\omega_{2}, \omega_{3}\right\}}^{\prime}=a_{\left\{\omega_{2}, \omega_{3}\right\}}$,

$$
\begin{aligned}
\mathcal{J}_{\omega_{2}}\left(\left\langle c, a^{\prime}\right\rangle\right) & =\mathcal{J}_{\omega_{2}}(c)+\mathcal{J}_{\omega_{2}}\left(a^{\prime}\right) \\
& =\mathcal{J}_{\omega_{2}}(c)+\mathcal{J}_{\omega_{2}}\left(a_{\left\{\omega_{2}, \omega_{3}\right\}}^{\prime}\right) \\
& =\mathcal{J}_{\omega_{2}}(c)+\mathcal{J}_{\omega_{2}}\left(a_{\left\{\omega_{2}, \omega_{3}\right\}}\right) \\
& =J_{\omega_{2}}(c)+\mathcal{J}_{\omega_{2}}(a) \\
& =\mathcal{J}_{\omega_{2}}(\langle c, a\rangle) .
\end{aligned}
$$

Similarly, $\mathcal{J}_{\omega_{3}}\left(\left\langle c, a^{\prime}\right\rangle\right)=\mathcal{J}_{\omega_{3}}(\langle c, a\rangle)$. To finish the proof, then, it suffices to show that $\mathcal{J}_{\omega_{1}}\left(\left\langle c, a^{\prime}\right\rangle\right)<\mathcal{J}_{\omega_{1}}(\langle c, a\rangle)$. To that end, use (1) to compute

$$
\mathcal{J}_{\omega_{1}}\left(v_{\omega_{1}}\right)=0
$$

and

$$
\mathcal{J}_{\omega_{1}}\left(v_{\omega_{2}}\right)=4
$$

So, using Temporal Separability and the definition of $\mathcal{J}$ on credal acts once more, we find

$$
\begin{aligned}
\mathcal{J}_{\omega_{1}}\left(\left\langle c, a^{\prime}\right\rangle\right) & =\mathcal{J}_{\omega_{1}}(c)+\mathcal{J}_{\omega_{1}}\left(v_{\omega_{1}}\right) \\
& <\mathcal{J}_{\omega_{1}}(c)+\mathcal{J}_{\omega_{1}}\left(v_{\omega_{2}}\right) \\
& =\mathcal{J}_{\omega_{1}}(\langle c, a\rangle) .
\end{aligned}
$$

We conclude that $\left\langle c, a^{\prime}\right\rangle$ weakly accuracy-dominates $\langle c, a\rangle$.

This completes the counterexample to (III).

Now, regarding the other points I have raised, I claim that the following theorem holds true and that its proof improves the one given by Briggs and Pettigrew.

Theorem 3. Assume that $\mathcal{J}$ satisfies Separability, Continuity, Extensionality, Strict Propriety, and Temporal Separability.

(a) Let $\langle c, a\rangle$ be a credal strategy. (1) If $c$ is not probabilistic, then there is a probabilistic and conditionalizing credal strategy that strongly accuracy-dominates $\langle c, a\rangle$. (2) If $c$ is probabilistic and $a_{E}$ is not probabilistic for some $E \in \mathcal{E}$ with $c(E)>0$, then there is a probabilistic and conditionalizing credal strategy that strongly accuracy-dominates $\langle c, a\rangle$. (3) If $c$ is probabilistic and $a_{E}$ is not probabilistic for some $E \in \mathcal{E}$ with $c(E)=0$, then there is a probabilistic and conditionalizing credal strategy that weakly accuracydominates $\langle c, a\rangle$. 
(b) For each credal strategy that is not conditionalizing, there is an alternative credal strategy that is probabilistic and conditionalizing that strongly accuracy-dominates it.

(c) For each credal strategy that is probabilistic and conditionalizing, (1) there is no alternative credal strategy whatsoever that strongly accuracy-dominates it, and (2) there is no non-conditionalizing credal strategy that even weakly accuracy-dominates it.

Proof. See Appendix A.

To illustrate some of the significance of Theorem 3, consider the following normative premise, which Briggs and Pettigrew use in their argument for conditionalization.

Normative Premise 1 for Credal Strategy Conditionalization It's impermissible for an agent to adopt a credal strategy $\langle c, a\rangle$ if there is another credal strategy that (i) strongly accuracy-dominates $\langle c, a\rangle$, and (ii) is not itself weakly accuracy-dominated (Briggs and Pettigrew, 2020, pp. 171-172).

This normative premise is the credal strategy analogue of the Normative Premise for Probabilism. Briggs and Pettigrew use it to argue for the following conclusion.

B \& $\mathbf{P}$ Conclusion Provided the inaccuracy measure satisfies the relevant properties, it's impermissible to adopt a credal strategy that is not conditionalizing. ${ }^{4}$

Unfortunately, in view of the fact that (III) is false, Briggs and Pettigrew's argument is unsound. Moreover, if we replace (III) with Theorem 3(c), the resulting argument from Normative Premise 1 for Credal Strategy Conditionalization to B \& P Conclusion is invalid. This is because, although every credal strategy that is not conditionalizing is strongly accuracy-dominated by a conditionalizing strategy, we cannot conclude that this conditionalizing strategy is not itself weakly accuracy-dominated. For some conditionalizing strategies are weakly accuracy-dominated, as we have seen in the Counterexample to (III). So, for all that has been shown, we cannot conclude that it is impermissible to adopt credal strategies that are not conditionalizing.

I will now suggest two ways to recover a sound argument for conditionalization. The first way is to adopt a different normative premise. The following, stronger normative premise combines with Theorem 3 to produce a sound argument for the B \& P Conclusion.

Normative Premise 2 for Credal Strategy Conditionalization It's impermissible for an agent to adopt a credal strategy $\langle c, a\rangle$ if there is another credal strategy that (i) strongly accuracy-dominates $\langle c, a\rangle$, and (ii) is not itself strongly accuracy-dominated.

It's noteworthy, however, that this premise is no longer analogous to the Normative Premise for Probabilism. The latter refers to weak accuracy-dominance in clause (ii), while Normative Premise 2 for Credal Strategy Conditionalization refers to strong accuracy-dominance in clause (ii). This difference between the two premises is not necessarily a problem, but, if one wished to defend both premises, it is a difference that would stand in need of explanation.

\footnotetext{
${ }^{4}$ This is a rephrasing of what Briggs and Pettigrew call "Plan Conditionalization (wide scope)" (p. 172). After presenting their argument for this thesis, Briggs and Pettigrew go on to investigate whether the argument also supports wide and narrow scope versions of what they call "Diachronic Conditionalization." I will not be assessing those investigations here.
} 
Why should the normative standard governing credences be different from the one governing credal strategies? Rather than pursue this question here, I will now suggest a second way of recovering a sound argument for conditionalization, which does not appeal to a normative premise that is different from Briggs and Pettigrew's.

Let us recall the problem with Normative Premise 1 for Credal Strategy Conditionalization. For all we have shown so far, there might be a credal strategy that is not conditionalizing and strongly accuracy-dominated only by probabilistic and conditionalizing strategies that are themselves weakly accuracy-dominated. In order for Normative Premise 1 for Credal Strategy Conditionalization to support the B \& P Conclusion, it needs to be the case that one can always find a conditionalizing strategy that strongly accuracy-dominates and is not itself weakly accuracy-dominated.

This, it turns out, can be done. ${ }^{5}$ What's more, we can characterize precisely the class of conditionalizing strategies that strongly dominate and are not weakly dominated. In order to do this, however, we need to introduce a new definition.

By way of motivating the new definition, let us recall the source of all the problems we have found with Briggs and Pettigrew's arguments: conditionalizations can behave in arbitrary and anomalous ways on propositions that have zero credence. In particular, the definition of conditionalization (3) allows $a_{E}(E) \neq 1$ when $c(E)=0$. But there is a strong precedent against allowing this. Indeed, in the literature on primitive conditional probability, it is usually taken as an axiom that the probability of $E$ given $E$ is $1 .^{6}$ This leads us to the following definition.

A credal act $a$ is Blackwell if for for all $E \in \mathcal{E}$ and all $\omega \notin E, a_{E}\{\omega\}=0$. In other words, Blackwell credal acts assign all of their credence to the proposition that is learned. Note that if $a_{E}$ is probabilistic for all $E \in \mathcal{E}$, then $a$ is Blackwell just in case $a_{E}(E)=1$ for all $E \in \mathcal{E} .^{7}$ That is, $a$ assigns credence 1 to the proposition that is learned. Say that a credal strategy $\langle c, a\rangle$ is Blackwell if $a$ is.

A further hint that this is the right definition to consider can be found in the Counterexample to (III). In that example, both $\langle c, a\rangle$ and $\left\langle c, a^{\prime}\right\rangle$ are probabilistic and conditionalizing, but the latter is Blackwell and the former is not. As we saw, the Blackwell strategy weakly accuracydominates the non-Blackwell one. Upon inspecting the example a bit more, one sees that the Blackwell strategy is not weakly accuracy-dominated. This insight from the counterexample generalizes to the following result.

Theorem 4. Assume that $\mathcal{J}$ satisfies Separability, Continuity, Extensionality, Strict Propriety, and Temporal Separability. Let $c$ be a probabilistic credence function, and let $\langle c, a\rangle$ be a conditionalizing strategy.

\footnotetext{
${ }^{5}$ In fact, a transfinite induction, much like the one given by Briggs and Pettigrew, establishes the result. The rough idea is that a transfinite sequence of conditionalizing strategies such that strategies later in the sequence weakly accuracy-dominate strategies later in the sequence must terminate eventually at a strategy that is not itself weakly-accuracy dominated. After all, there are far fewer conditionalizing strategies than ordinal numbers. As before, I avoid this fancy machinery because it's not needed.

${ }^{6}$ See, for example, Popper (1959); Renyi (1970); Dubins (1975); Hájek (2003).

${ }^{7}$ I would have preferred to call Blackwell credal acts "proper credal acts," adopting a standard terminology from probability theory, but this would conflict with the standard terminology for inaccuracy measures. Instead, I have followed Meehan and Zhang (2020) in using a term that honors David Blackwell, who made seminal contributions to the study of probabilistic properness (Blackwell and Ryll-Nardzewski, 1963; Blackwell and Dubins, 1975).
} 
(a) If $\langle c, a\rangle$ is not Blackwell, then it is weakly accuracy-dominated by a credal strategy that is probabilistic, conditionalizing, and Blackwell.

(b) Conversely, if $\langle c, a\rangle$ is Blackwell and probabilistic, then it is not weakly accuracydominated by any credal strategy whatsoever.

Proof. See Appendix B.

Theorem 4, together with our earlier results, allows us to derive the $\mathrm{B} \& \mathrm{P}$ conclusion from Normative Premise 1 for Credal Strategy Conditionalization, thereby obviating the explanatory burden incurred by adopting the stronger Normative Premise 2 for Credal Strategy Conditionalization. Indeed, under the assumption that the inaccuracy measure satisfies the relevant properties, if a credal strategy is not conditionalizing, then Theorem 3(b) says that it is strongly accuracy-dominated by a credal strategy that is probabilistic and conditionalizing. By Theorem 4(a), we can assume that the strongly dominating strategy is Blackwell. ${ }^{8}$ By Theorem 4(b), the strongly dominating strategy is not itself weakly accuracy-dominated. Therefore, by Normative Premise 1 for Credal Strategy Conditionalization, it is impermissible to adopt the strategy that is not conditionalizing. This is the desired B \& $\mathrm{P}$ conclusion.

There are two more philosophical points to make before concluding this section. The first begins by noting another puzzling feature of Briggs and Pettigrew's argument for conditionalization, which Theorem 3 can help to address. Observe that the B \& $\mathrm{P}$ conclusion asserts that it's impermissible to adopt a credal strategy that is not conditionalizing, but it doesn't assert that it's impermissible to adopt a credal strategy that is not probabilistic. And, indeed, Briggs and Pettigrew's (I) is too weak to support this additional conclusion, since it shows only that non-probabilistic credal strategies are weakly accuracy-dominated. So, for all that the Briggs and Pettigrew argument shows, it is permissible to adopt non-probabilistic credal strategies as long as they are conditionalizing. That looks like a gap worth filling.

Here, the added strength of Theorem 3(a) is useful. Note how it improves Briggs and Pettigrew's (I): in Theorem 3(a), three cases are considered in which $\langle c, a\rangle$ is not probabilistic, and in all but one it is shown that $\langle c, a\rangle$ is strongly accuracy-dominated by a strategy that is probabilistic and conditionalizing. As above, we can assume that the strongly dominating strategy is Blackwell, by Theorem 4(a). And, by Theorem 4(b), it follows that the strongly dominating strategy is not itself weakly accuracy-dominated. Thus, combining our results with Normative Premise 1 for Credal Strategy Conditionalization, we get:

Probabilistic Conclusion Provided the inaccuracy measure satisfies the relevant properties, it's impermissible to adopt a credal strategy $\langle c, a\rangle$ if $c$ is not probabilistic or $a_{E}$ is not probabilistic for some $E$ such that $c(E)>0$.

The final point is that we can derive a conclusion that is stronger than the B \& P Conclusion, provided we are willing to accept the following strengthening of Normative Premise 1 for Credal Strategy Conditionalization.

Normative Premise 3 for Credal Strategy Conditionalization It's impermissible for an agent to adopt a credal strategy $\langle c, a\rangle$ if there is another credal strategy that (i) weakly accuracy-dominates $\langle c, a\rangle$, and (ii) is not itself weakly accuracy-dominated.

\footnotetext{
${ }^{8}$ If it is not, then it is weakly accuracy-dominated by a probabilistic, conditionalizing strategy that is Blackwell, and this strategy also strongly accuracy-dominates the one that is not conditionalizing.
} 
Together with Theorems 3 and 4, Normative Premise 3 for Credal Strategy Conditionalization entails the following conclusion.

Blackwell Conclusion Provided the inaccuracy measure satisfies the relevant properties, it's impermissible to adopt a credal strategy that isn't probabilistic, conditionalizing, and Blackwell.

This is because, under the assumption that the inaccuracy measure satisfies the relevant properties, if a credal strategy is either not probabilistic or not conditionalizing, then it is weakly accuracy-dominated by a credal strategy that is probabilistic and conditionalizing, by Theorem 3(a) and (b). By Theorem 4(a), we can assume that the weakly dominating strategy is Blackwell, and Theorem 4(b) implies that this strategy is not itself weakly accuracydominated. Normative Premise 3 for Credal Strategy Conditionalization then implies that it's impermissible to adopt the original credal strategy. Moreover, if the original credal strategy is probabilistic and conditionalizing, but not Blackwell, then, by Theorem 4, it is weakly accuracy-dominated by a credal strategy with all of these properties, which, in turn, is not itself weakly-accuracy dominated. So, Normative Premise 3 for Credal Strategy Conditionalization implies that it is impermissible to adopt the original credal strategy. We thereby reach the Blackwell Conclusion.

As with Normative Premise 2 for Credal Strategy Conditionalization, Normative Premise 3 for Credal Strategy Conditionalization is not analogous to the Normative Premise for Probabilism. The latter refers to strong accuracy-dominance in clause (i), while the former refers to weak accuracy-dominance in clause (i). So, once again, should one wish to defend both premises, this difference between them would stand in need of explanation.

\section{Summary}

I began by arguing that philosophers interested in epistemic decision theory should care about whether the mathematical premises used in the theory's arguments are true, strong, and straightforward to prove. My analysis of Briggs and Pettigrew's theorem supports this position.

First, I showed that one of the claims in Briggs and Pettigrew's theorem is false and therefore cannot serve as a premise in a sound argument for conditionalization. I suggested two ways of repairing their argument. The first was to adopt a stronger normative premise (Normative Premise 2 for Credal Strategy Conditionalization). The second was to derive stronger mathematical results (Theorem 4) that combine with Briggs and Pettigrew's original normative premise to produce a sound argument for their conclusion.

Second, I strengthened another claim in Briggs and Pettigrew's theorem and used the strengthened claim (Theorem 3(a)) to support a natural conclusion that doesn't appear in Briggs and Pettigrew's paper, namely that credal strategies should be probabilistic (Probabilistic Conclusion).

Third, I used my stronger results to present an argument that credal strategies should be probabilistic, conditionalizing, and Blackwell (Blackwell Conclusion), a conclusion stronger than the one supported by Briggs and Pettigrew's argument. I have left open, however, whether the normative premise on which this argument relies (Normative Premise 3 for Credal Strategy Conditionalization) is defensible. 
My final contribution will be to show that Briggs and Pettigrew's proofs can be simplified considerably. Readers who are interested in this final point are invited to continue to the appendices. 


\section{Appendix A}

Theorem 3. Assume that $\mathcal{J}$ satisfies Separability, Continuity, Extensionality, Strict Propriety, and Temporal Separability.

(a) Let $\langle c, a\rangle$ be a credal strategy. (1) If $c$ is not probabilistic, then there is a probabilistic and conditionalizing credal strategy that strongly accuracy-dominates $\langle c, a\rangle$. (2) If $c$ is probabilistic and $a_{E}$ is not probabilistic for some $E \in \mathcal{E}$ with $c(E)>0$, then there is a probabilistic and conditionalizing credal strategy that strongly accuracy-dominates $\langle c, a\rangle$. (3) If $c$ is probabilistic and $a_{E}$ is not probabilistic for some $E \in \mathcal{E}$ with $c(E)=0$, then there is a probabilistic and conditionalizing credal strategy that weakly accuracydominates $\langle c, a\rangle$.

(b) For each credal strategy that is not conditionalizing, there is an alternative credal strategy that is probabilistic and conditionalizing that strongly accuracy-dominates it.

(c) For each credal strategy that is probabilistic and conditionalizing, (1) there is no alternative credal strategy whatsoever that strongly accuracy-dominates it, and (2) there is no non-conditionalizing credal strategy that even weakly accuracy-dominates it.

\section{Proof of Theorem 3}

Assume throughout that $\mathcal{J}$ satisfies Separability, Continuity, Extensionality, Strict Propriety, and Temporal Separability.

The proofs of parts (a) and (b) rely on the following lemma, which builds on the observations about arbitrary behavior on zero credence propositions that are expressed in Lemma 1 .

Lemma 2. Let $c$ be a probabilistic credence function, and let $\langle c, a\rangle$ be a conditionalizing strategy. If $\langle c, a\rangle$ is not probabilistic, then it is weakly accuracy-dominated by a credal strategy $\left\langle c, a^{\prime}\right\rangle$ that is both probabilistic and conditionalizing.

Proof. If $\langle c, a\rangle$ is not probabilistic, then $a_{E}$ is not probabilistic for at least one $E \in \mathcal{E}$. For all such $E, c(E)=0$, by Lemma 1(ii); and, by Theorem 1, there is a probabilistic credence function $p_{E}$ that strongly accuracy-dominates $a_{E}$. For all $E \in \mathcal{E}$, define the credal act $a^{\prime}$ by

$$
a_{E}^{\prime}=\left\{\begin{array}{l}
a_{E} \text { if } a_{E} \text { is probabilistic } \\
p_{E} \text { otherwise }
\end{array}\right.
$$

Then, $a^{\prime}$ is probabilistic and weakly accuracy-dominates $a$. By Lemma 1(i), $a^{\prime}$ is a conditionalization of $c$ because it differs from $a$ only on propositions with zero $c$-credence. By Temporal Separability, $\left\langle c, a^{\prime}\right\rangle$ weakly accuracy-dominates $\langle c, a\rangle$.

We now give the proof of Theorem 3. It will be convenient to prove (b) first.

Proof of Theorem 3 (b). The argument follows the one for (II) given by Briggs and Pettigrew (2020), which, in turn, relies on some technical propositions in Predd et al. (2009). My aim here is simply to emphasize that the last step of Briggs and Pettigrew's argument (7.4, pp. 178-179) involving a transfinite induction can be eliminated. I will state some lemmas that 
summarize the key steps of Briggs and Pettigrew's argument. But first we need to introduce some notation.

Let $\mathcal{A}=\left\{A_{1}, \ldots, A_{m}\right\}$. Note that every credence function can be identified with a vector in $\mathbb{R}^{m}$ in the following way:

$$
c=\left\langle c\left(A_{1}\right), \ldots, c\left(A_{m}\right)\right\rangle .
$$

Similarly, with $\mathcal{E}=\left\{E_{1}, \ldots, E_{n}\right\}$, every credal strategy $\langle c, a\rangle$ can be identified with a vector in $\mathbb{R}^{m+m n}$ :

$$
\langle c, a\rangle=\langle\underbrace{c\left(A_{1}\right), \ldots, c\left(A_{m}\right)}_{c}, \underbrace{a_{E_{1}}\left(A_{1}\right), \ldots, a_{E_{1}}\left(A_{m}\right)}_{a_{E_{1}}}, \ldots, \underbrace{a_{E_{n}}\left(A_{1}\right), \ldots, a_{E_{n}}\left(A_{m}\right)}_{a_{E_{n}}}\rangle .
$$

Now, given a credal strategy $\langle c, a\rangle$ and $\omega \in \Omega$, define $\langle c, a\rangle_{\omega}$ by replacing both $c$ and $a_{\mathcal{E}_{\omega}}$ with the valuation function $v_{\omega}$ in the vector representation of $\langle c, a\rangle$. More formally, let $i_{\omega}$ be that $i \in\{1, \ldots, n\}$ for which $\mathcal{E}_{\omega}=E_{i}$, i.e. that $i$ for which $\omega \in E_{i}$, and define

$$
\begin{aligned}
\langle c, a\rangle_{\omega}=\langle & \langle\underbrace{v_{\omega}\left(A_{1}\right), \ldots, v_{\omega}\left(A_{m}\right)}_{v_{\omega} \text { replacing } c}, \ldots, a_{E_{i_{\omega}-1}}\left(A_{1}\right), \ldots, a_{E_{i_{\omega}-1}}\left(A_{m}\right), \underbrace{v_{\omega}\left(A_{1}\right), \ldots, v_{\omega}\left(A_{m}\right)}_{v_{\omega} \text { replacing } a_{\mathcal{E}_{\omega}}}, \\
& \left.a_{E_{i_{\omega}+1}}\left(A_{1}\right), \ldots, a_{E_{i_{\omega}+1}}\left(A_{m}\right), \ldots, a_{E_{n}}\left(A_{1}\right), \ldots, a_{E_{n}}\left(A_{m}\right)\right\rangle .
\end{aligned}
$$

Briggs and Pettigrew show the following.

Lemma 3 (Briggs and Pettigrew (2020), Lemma 2). Let $\langle c, a\rangle$ be a credal strategy. Then, $c$ is probabilistic and $\langle c, a\rangle$ is a conditionalizing strategy if and only if $\langle c, a\rangle$ is in the convex hull of $\left\{\langle c, a\rangle_{\omega}: \omega \in \Omega\right\}$.

Lemma 4 (Briggs and Pettigrew (2020), p. 178). If the credal strategy $\langle c, a\rangle$ is not in the convex hull of $\left\{\langle c, a\rangle_{\omega}: \omega \in \Omega\right\}$, then there is some credal strategy $\left\langle c^{\prime}, a^{\prime}\right\rangle$ in the convex hull of $\left\{\langle c, a\rangle_{\omega}: \omega \in \Omega\right\}$ such that

$$
\mathcal{J}_{\omega}\left(\left\langle c^{\prime}, a^{\prime}\right\rangle\right)<\mathcal{J}_{\omega}(\langle c, a\rangle)
$$

for all $\omega \in \Omega$.

So, if $\langle c, a\rangle$ is a credal strategy that is not conditionalizing, then, by Lemma $3,\langle c, a\rangle$ is not in the convex hull of $\left\{\langle c, a\rangle_{\omega}: \omega \in \Omega\right\}$. By Lemma $4,\langle c, a\rangle$ is strongly accuracy-dominated by a credal strategy $\left\langle c^{\prime}, a^{\prime}\right\rangle$ that is in the convex hull of $\left\{\langle c, a\rangle_{\omega}: \omega \in \Omega\right\}$. Calling on Lemma 3 once more, we find that $c^{\prime}$ is probabilistic and $\left\langle c^{\prime}, a^{\prime}\right\rangle$ is a conditionalizing strategy. If $\left\langle c^{\prime}, a^{\prime}\right\rangle$ is probabilistic, then we are done. If not, apply Lemma 2 to find a credal strategy $\left\langle c^{\prime \prime}, a^{\prime \prime}\right\rangle$ that is probabilistic and conditionalizing and weakly accuracy-dominates $\left\langle c^{\prime}, a^{\prime}\right\rangle$. Then, $\left\langle c^{\prime \prime}, a^{\prime \prime}\right\rangle$ strongly accuracy-dominates $\langle c, a\rangle$.

Proof of Theorem 3 (a). (1) If $c$ is not probabilistic, then the argument proceeds as in the proof of (b) above: by Lemmas 3 and $4,\langle c, a\rangle$ is strongly accuracy-dominated by a credal strategy $\left\langle c^{\prime}, a^{\prime}\right\rangle$ such that $c^{\prime}$ is probabilistic and $\left\langle c^{\prime}, a^{\prime}\right\rangle$ is a conditionalizing strategy; if $\left\langle c^{\prime}, a^{\prime}\right\rangle$ is not already probabilistic, then, by Lemma 2 , it is weakly accuracy-dominated by a probabilistic and conditionalizing strategy $\left\langle c^{\prime \prime}, a^{\prime \prime}\right\rangle$, and $\left\langle c^{\prime \prime}, a^{\prime \prime}\right\rangle$ strongly accuracy-dominates $\langle c, a\rangle$.

(2) If $c$ is probabilistic and $a_{E}$ is not probabilistic for some $E \in \mathcal{E}$ with $c(E)>0$, then $\langle c, a\rangle$ is not a conditionalizing strategy, by Lemma 1(ii). By part (b), $\langle c, a\rangle$ is strongly accuracy-dominated by a credal strategy that is probabilistic and conditionalizing. 
(3) Suppose that $c$ is probabilistic and $a_{E}$ is not probabilistic for some $E \in \mathcal{E}$ with $c(E)=0$. If $\langle c, a\rangle$ is not a conditionalizing strategy, then it is strongly accuracy-dominated by (b), so assume that $\langle c, a\rangle$ is a conditionalizing strategy. Then, by Lemma $2,\langle c, a\rangle$ is weakly accuracy-dominated.

Proof of Theorem 3(c). Assume that $\langle c, a\rangle$ is probabilistic and conditionalizing. Using Temporal Separability, we can compute the $c$-expected inaccuracy of $\langle c, a\rangle$ :

$$
\exp _{c}(\mathcal{J}(\langle c, a\rangle))={ }_{d f} \sum_{\omega \in \Omega} c\{\omega\} \mathcal{J}_{\omega}(\langle c, a\rangle)=\sum_{\omega \in \Omega} c\{\omega\} \mathcal{J}_{\omega}(c)+\exp _{c}(\mathcal{J}(a))
$$

For (1), use Strict Propriety and Theorem 2 to observe that

$$
\sum_{\omega \in \Omega} c\{\omega\} \mathcal{J}_{\omega}(c)+\exp _{c}(\mathcal{J}(a)) \leq \sum_{\omega \in \Omega} c\{\omega\} \mathcal{J}_{\omega}\left(c^{\prime}\right)+\exp _{c}\left(\mathcal{J}\left(a^{\prime}\right)\right)
$$

for all credal strategies $\left\langle c^{\prime}, a^{\prime}\right\rangle$. But if $\left\langle c^{\prime}, a^{\prime}\right\rangle$ strongly accuracy-dominates $\langle c, a\rangle$, so that $\mathcal{J}_{\omega}\left(\left\langle c^{\prime}, a^{\prime}\right\rangle\right)<\mathcal{J}_{\omega}(\langle c, a\rangle)$ for all $\omega \in \Omega$, then

$$
\exp _{c}\left(\mathcal{J}\left(\left\langle c^{\prime}, a^{\prime}\right\rangle\right)\right)<\exp _{c}(\mathcal{J}(\langle c, a\rangle)) \text {. }
$$

So, no probabilistic and conditionalizing credal strategy is strongly accuracy-dominated. ${ }^{9}$

For (2), suppose there were a non-conditionalizing credal strategy $\left\langle c^{\prime}, a^{\prime}\right\rangle$ that weakly accuracy-dominated $\langle c, a\rangle$. By part (b) of the theorem, $\left\langle c^{\prime}, a^{\prime}\right\rangle$ would be strongly accuracydominated, and therefore so would $\langle c, a\rangle$, contradicting what we have just shown.

\section{Appendix B}

Theorem 4. Assume that $\mathcal{J}$ satisfies Separability, Continuity, Extensionality, Strict Propriety, and Temporal Separability. Let $c$ be a probabilistic credence function, and let $\langle c, a\rangle$ be a conditionalizing strategy.

(a) If $\langle c, a\rangle$ is not Blackwell, then it is weakly accuracy-dominated by a credal strategy that is probabilistic, conditionalizing, and Blackwell.

(b) Conversely, if $\langle c, a\rangle$ is Blackwell and probabilistic, then it is not weakly accuracydominated by any credal strategy whatsoever.

\section{Proof of Theorem 4}

Proof of Theorem 4(a). By Lemma 2, we can assume without loss of generality that $\langle c, a\rangle$ is probabilistic. If $\langle c, a\rangle$ is not Blackwell, then there is some subset $\left\{E_{1}, \ldots, E_{k}\right\}$ of $\mathcal{E}$ such that $c\left(E_{i}\right)=0$ and $a_{E_{i}}\left(E_{i}\right)<1$ for all $i \in\{1, \ldots, k\}$. For each $i \in\{1, \ldots, k\}$, it follows that $a_{E_{i}}$ is

\footnotetext{
${ }^{9}$ The argument given here is similar to the one that Briggs and Pettigrew give in their proof of (III). The problem with their argument is that it makes the following fallacious inference: if a credal strategy is an expected inaccuracy minimizer, then it is not weakly accuracy-dominated. But, as we have seen, conditionalizations are both expected inaccuracy minimizers (Theorem 2) and capable of being weakly accuracydominated by other conditionalizations when propositions in the evidence partition have zero credence, as in the Counterexample to (III).
} 
not in the convex hull of $\left\{v_{w}: w \in E_{i}\right\}$, which in turn implies that there is some $p_{i}$ in the convex hull of $\left\{v_{w}: w \in E_{i}\right\}$ such that

$$
\mathcal{J}_{\omega}\left(p_{i}\right)<\mathcal{J}_{\omega}\left(a_{E_{i}}\right)
$$

for all $\omega \in E_{i}$ (the proof of this latter fact is exactly like Briggs and Pettigrew's proof of Lemma 4 and is omitted). Since $p_{i}$ is in the convex hull of $\left\{v_{w}: w \in E_{i}\right\}$, it is probabilistic and $p_{i}\left(E_{i}\right)=1$. Now define a new credal act $a^{\prime}$ by

$$
a_{E}^{\prime}=\left\{\begin{array}{l}
p_{i} \text { if } E=E_{i} \text { for some } i \in\{1, \ldots k\} \\
a_{E} \text { otherwise }
\end{array}\right.
$$

By Lemma 1(i), $\left\langle c, a^{\prime}\right\rangle$ is conditionalizing, and, by construction, it is probabilistic and Blackwell. By Temporal Separability and (7), if $\omega \in \bigcup_{i=1}^{k} E_{i}$, then, for some $i \in\{1, \ldots, k\}$,

$$
\mathcal{J}_{\omega}\left(\left\langle c, a^{\prime}\right\rangle\right)=\mathcal{J}_{\omega}(c)+\mathcal{J}_{\omega}\left(p_{i}\right)<\mathcal{J}_{\omega}(c)+\mathcal{J}_{\omega}\left(a_{E_{i}}\right)=\mathcal{J}_{\omega}(\langle c, a\rangle)
$$

otherwise $\mathcal{J}_{\omega}\left(\left\langle c, a^{\prime}\right\rangle\right)=\mathcal{J}_{\omega}(\langle c, a\rangle)$. So $\left\langle c, a^{\prime}\right\rangle$ weakly accuracy-dominates $\langle c, a\rangle$.

Proof of Theorem $4(b)$. Suppose that $\langle c, a\rangle$ is probabilistic and weakly accuracy-dominated by $\left\langle c^{\prime}, a^{\prime}\right\rangle$. We aim to show that $\langle c, a\rangle$ is not Blackwell, that is, that $a_{E}(E)<1$ for some $E \in \mathcal{E}$.

We begin by claiming that $c=c^{\prime}$. Indeed, due to the weak accuracy-dominance, we have

$$
\exp _{c}\left(\mathcal{J}\left(\left\langle c^{\prime}, a^{\prime}\right\rangle\right)\right) \leq \exp _{c}(\mathcal{J}(\langle c, a\rangle))
$$

But Theorem 2 implies that $\exp _{c}\left(\mathcal{J}\left(a^{\prime}\right)\right) \geq \exp _{c}(\mathcal{J}(a))$, so

$$
\sum_{\omega \in \Omega} c\{\omega\} \mathcal{J}_{\omega}\left(c^{\prime}\right) \leq \sum_{\omega \in \Omega} c\{\omega\} \mathcal{J}_{\omega}(c)
$$

which implies that $c=c^{\prime}$, by Strict Propriety.

Weak accuracy-dominance now implies that $a \neq a^{\prime}$, so $a_{E} \neq a_{E}^{\prime}$ for some $E \in \mathcal{E}$. It also implies, together with Temporal Separability, that $\mathcal{J}_{\omega}\left(a^{\prime}\right) \leq \mathcal{J}_{\omega}(a)$ for all $\omega \in \Omega$. In particular, $\mathcal{J}_{\omega}\left(a_{E}^{\prime}\right) \leq \mathcal{J}_{\omega}\left(a_{E}\right)$ for all $\omega \in E$, and therefore

$$
\sum_{\omega \in E} a_{E}\{\omega\} \mathcal{J}_{\omega}\left(a_{E}^{\prime}\right) \leq \sum_{\omega \in E} a_{E}\{\omega\} \mathcal{J}_{\omega}\left(a_{E}\right)
$$

If $a_{E}(E)=1$, then, because $a_{E}$ is probabilistic, (8) implies

$$
\sum_{\omega \in \Omega} a_{E}\{\omega\} \mathcal{J}_{\omega}\left(a_{E}^{\prime}\right) \leq \sum_{\omega \in \Omega} a_{E}\{\omega\} \mathcal{J}_{\omega}\left(a_{E}\right)
$$

And it follows from Strict Propriety that $a_{E}^{\prime}=a_{E}$, which is false. Thus, $a_{E}(E)<1$, and $\langle c, a\rangle$ is not Blackwell. 


\section{References}

Armendt, B. (1992). Dutch strategies for diachronic rules: When believers see the sure loss coming. In PSA: Proceedings of the biennial meeting of the Philosophy of Science Association, Number 1, pp. 217-229. Philosophy of Science Association.

Blackwell, D. and L. E. Dubins (1975). On existence and non-existence of proper, regular, conditional distributions. The Annals of Probability 3(5), 741-752.

Blackwell, D. and C. Ryll-Nardzewski (1963). Non-existence of everywhere proper conditional distributions. Annals of Mathematical Statistics 34(1), 223-235.

Briggs, R. and R. Pettigrew (2020). An accuracy-dominance argument for conditionalization. Noûs 54(1), 162-181.

Carr, J. R. (2019). A modesty proposal. Synthese.

Christensen, D. (1991). Clever bookies and coherent beliefs. The Philosophical Review 100(2), 229247.

Christensen, D. (1996). Dutch-book arguments depragmatized: Epistemic consistency for partial believers. The Journal of Philosophy 93(9), 450-479.

Dubins, L. E. (1975). Finitely additive conditional probabilities, conglomerability and disintegrations. The Annals of Probability 3(1), 89-99.

Gallow, J. D. (2019). Updating for externalists. Noûs.

Greaves, H. and D. Wallace (2006). Justifying conditionalization: Conditionalization maximizes expected epistemic utility. Mind 115(459), 607-632.

Hájek, A. (2003). What conditional probability could not be. Synthese 137(3), 273-323.

Lewis, D. (1999). Why conditionalize? In Papers in Metaphysics and Epistemology, Volume 2, pp. 403-407. Cambridge University Press.

Meehan, A. and S. Zhang (2020). Kolmogorov conditionalizers can be Dutch booked. Review of Symbolic Logic, forthcoming.

Popper, K. (1959). The Logic of Scientific Discovery. Hutchinson \& Co.

Predd, J. B., R. Seiringer, E. H. Lieb, D. N. Osherson, H. V. Poor, and S. R. Kulkarni (2009). Probabilistic coherence and proper scoring rules. IEEE Transactions on Information Theory 55(10), 4786-4792.

Renyi, A. (1970). Foundations of Probability. Holden Day.

Schoenfield, M. (2017). Conditionalization does not (in general) maximize expected accuracy. Mind 126(504), 1155-1187. 